\title{
Severe Dumping Syndrome in a 6-year-old Girl with Congenital Microgastria Treated by Hunt-Lawrence Pouch
}

\author{
Claudia Filisetti $^{1,2}$ Luciano Maestri ${ }^{1}$ Milena Meroni ${ }^{1}$ Federica Marinoni ${ }^{1}$ Giovanna Riccipetitoni ${ }^{1}$ \\ ${ }^{1}$ Department of Pediatric Surgery, "V. Buzzi” Children Hospital, \\ Milano, Italy \\ 2 University of Pavia, PhD School, Pavia, Italy \\ Address for correspondence Claudia Filisetti, Department of \\ Pediatric Surgery, “V. Buzzi” Children Hospital, Via Castelvetro, \\ Milano 20154, Italy (e-mail: claudiafilisetti@yahoo.it).
}

Eur J Pediatr Surg Rep 2017;5:e17-e20.

\begin{abstract}
Keywords

- congenital microgastria

- dumping syndrome

- Hunt-Lawrence pouch

Microgastria is a rare congenital condition often associated with other anomalies. In the present report we describe the case of a 6-year-old girl with isolated CM who presented with dumping syndrome successfully treated by a Hunt-Lawrence pouch.
\end{abstract}

\section{New Insights and the Importance for the Pediatric Surgeon}

The case we present is relevant because it analyzes a new clinical situation (dumping syndrome) that occurs in a patient with congenital microgastria treated successfully with Hunt-Lawrence pouch.

\section{Introduction}

Congenital microgastria $(\mathrm{CM})$ is a disorder in fetal development leading to a tubular stomach with a small functional capacity. It was reported for the first time by Dide in 1894, but only in 1971 Schulz and Niemann described $\mathrm{CM}$ as a clinical condition. ${ }^{1,2} \mathrm{CM}$ is caused by an arrest in the embryogenesis of the dorsal mesogastrium during the fifth week of fetal development and is often located in a constellation of other anomalies. In a very small group of patients, $\mathrm{CM}$ represents an isolated malformation. ${ }^{2,3}$ The micro-stomach leads to a series of symptoms and signs such as gastroesophageal reflux, vomiting, aspiration, malnutrition, and failure to thrive. Dumping syndrome has been described by Anderson and Guzzetta as a consequence of a gastric augmentation in two cases of CM, but it has never been reported as the presenting symptom in a patient affected by $\mathrm{CM}^{4}$ We report on a 6 -year-old girl with $\mathrm{CM}$ and severe dumping syndrome who was treated by Hunt-Lawrence pouch (HLP).

received

November 24, 2016 accepted after revision February 14, 2017

\section{Case Report}

A 6-year-old girl was referred to our unit with a history of failure to thrive and frequent episodes of postprandial nausea associated with symptoms and signs related to severe hypoglycemia: dizziness, tachycardia, and decreased consciousness. Her weight was $13 \mathrm{~kg}$ and her height was $103 \mathrm{~cm}$, both under the third percentile. She was born at term in another institution after a pregnancy characterized by ultrasonographic findings of absent stomach and polyhydramnios; therefore esophageal atresia had been suspected but not confirmed at birth. In consideration of the prenatal finding, the patient was subjected to an upper gastrointestinal contrast study that showed a small stomach with a normal transit of the contrast to the small bowel. No other associated anomalies were detected. Initial conservative treatment with small and frequent meals was started, but the patient was lost to follow-up. Her parents reported that she progressively developed food aversion and symptoms

\footnotetext{
(c) 2017 Georg Thieme Verlag KG Stuttgart · New York
}

License terms

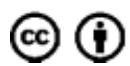

10.1055/s-0037-1601305. ISSN 2194-7619. 


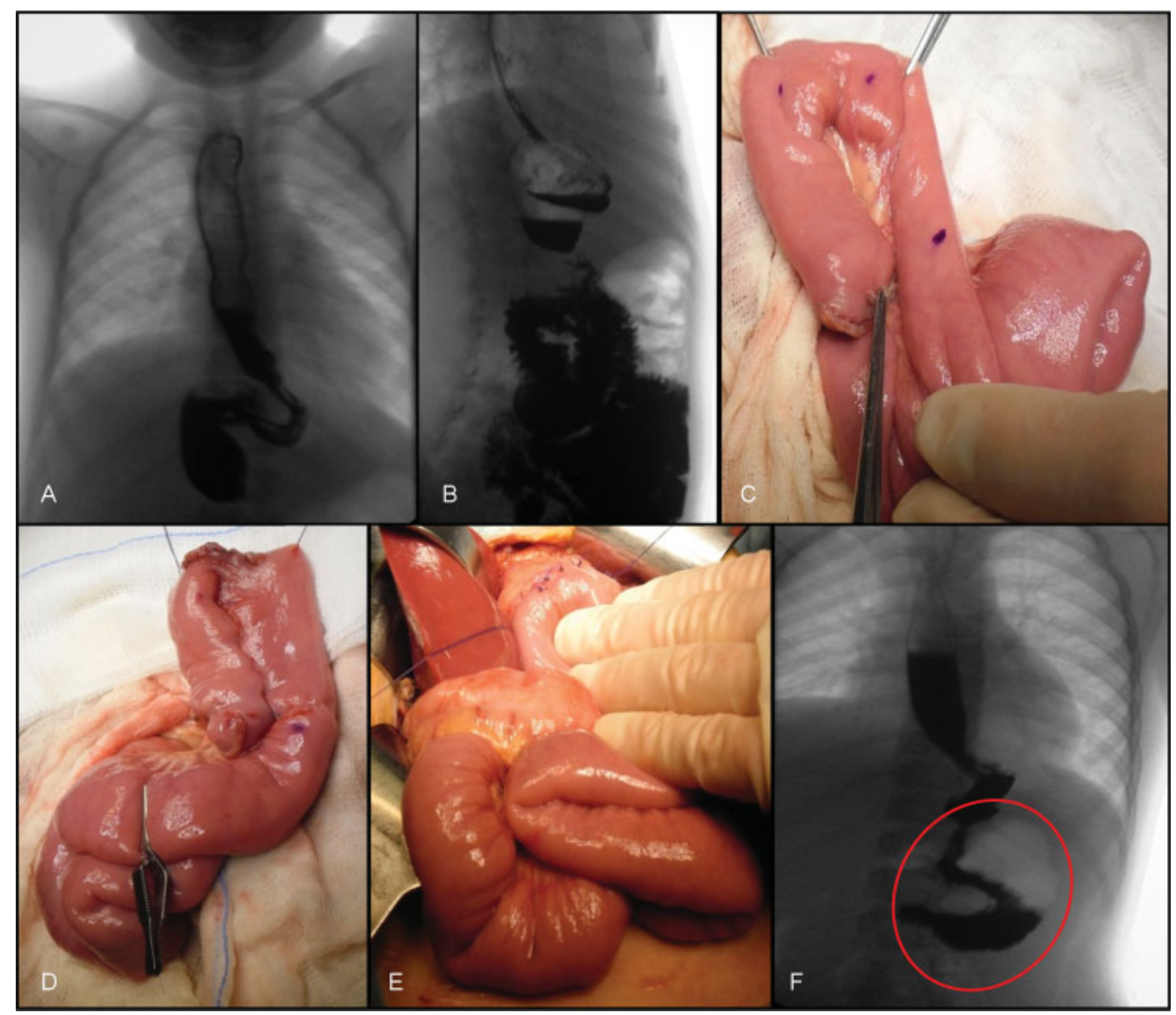

Fig. 1 Preoperative barium meal (A, B) demonstrates a micro-stomach and a very rapid transit in the small bowel. (C-E) Hunt-Lawrence pouch. Isolation of the jejunal tract (C), preparation of the jejunal pouch (D), and gastrojejunal anastomosis (E). Barium meal performed 11 days after surgery (F) demonstrating the absence of leakage or obstruction of the jejunal pouch (red circle).

such as dizziness, tachycardia, and frequent episodes of decreased consciousness, all referable to a late dumping syndrome. On our clinical examination the girl was very thin, short, and pale. All blood tests were normal, but 90 minutes after meals we detected hypoglycemia. We repeated a barium meal study that showed a normal esophagus, a very small tubular stomach with a very rapid transit of the contrast to the small bowel ( $\sim$ Fig. 1A-C). We decided to perform an HLP with a nutritional catheter jejunostomy ( - Fig. 1C-E, - Fig. 2). The postoperative course was uneventful, and the feeding was initiated 12 days after the operation. The child was discharged 3 weeks after surgery on normal oral feeding and enteral nutrition at night. Over the next 6 months of follow-up, the patient's weight and height increased from the 3rd to the 50th percentile and the jejunostomy tube could be removed. Eighteen months after the operation, no hypoglycemia or hyperglycemia episodes occurred; weight and height remained stable at the 50th percentile.

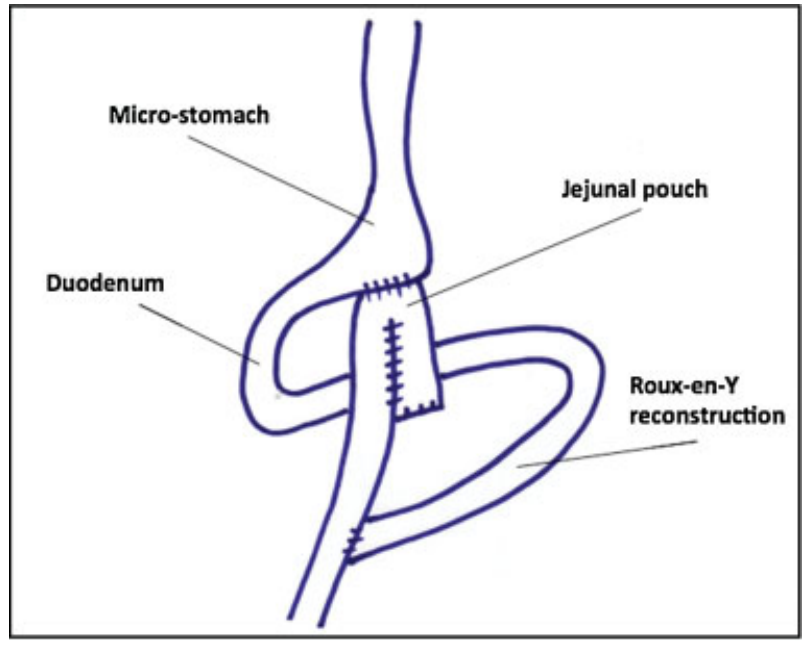

Fig. 2 Scheme of the Hunt-Lawrence pouch technique. 


\section{Discussion}

Although there are more than 60 reports on CM, the best treatment for this condition remains controversial. The reasons for this include an extremely variable clinical presentation and a frequent association of $\mathrm{CM}$ with other conditions. Only five cases of isolated CM are reported, ${ }^{5-9}$ and in these patients the clinical scenario is characterized by gastroesophageal reflux and the presence of feeding difficulties. All the other cases of $\mathrm{CM}$ reported are associated with additional anomalies. ${ }^{10,11}$ This clinical heterogeneity makes an objective evaluation of the therapeutic options problematic. Technically, the surgical approach (HLP) has been recognized anecdotally as safe and feasible in all the reported cases ( - Fig. 2), but its indications and timing of application have not been completely defined. ${ }^{5-10,12}$

Isolated CM is followed by symptoms and signs, including postprandial vomiting, growth retardation, failure to thrive, malnutrition, and recurrent aspiration pneumonia. All the reported cases have been treated with various maneuvers for nutrition and growth before a definitive operative intervention such as gastrojejunostomy, ${ }^{13}$ jejunostomy, ${ }^{11}$ construction of a spit fistula, ${ }^{14}$ or a Roux-en-Y feeding jejunostomy. ${ }^{11}$ All these treatments for CM are reported to be generally unsuccessful due to poor physical growth. Our patient was afflicted by dumping syndrome as the main clinical feature. Dumping syndrome is a well-known complication in children and adults after gastric surgery, and is characterized by typical signs and symptoms associated with postprandial hypoglycemia. Even now, the whole mechanism involved in dumping syndrome is not completely understood even though its pathophysiology (in the isolated $\mathrm{CM}$ ) probably has the same features of the late dumping syndrome that can develop in patients after gastric surgery. ${ }^{15}$ Under normal conditions the stomach acts as a reservoir, initiates digestion, and releases its contents in a controlled fashion. In CM there is a profound alteration in gastric emptying as a result of decreased volume with alteration of motility. In our patient the main symptom was a relevant postprandial hypoglycemia. The pivotal role of humoral factors in the pathogenesis of dumping has been demonstrated experimentally. ${ }^{16-18} \mathrm{An}$ increase in glucagon-like peptide-1 (GLP-1) secretion and the abnormal stimulation of insulin release seem to be crucial in the pathogenesis of severe hypoglycemia. After gastric surgery, dumping occurs as a complication. In our case the dumping has a similar pathophysiology with fast gastric emptying and the same response to the rapid transit of food. We decided to treat our patient with the HLP procedure. The procedure proved to be effective in treating all the signs and symptoms associated with dumping syndrome, immediately improving the quality of life of the patient. In the literature, HLP has been reported to be an operative treatment allowing amelioration of the nutritional prognosis. ${ }^{3}$ Nevertheless, the final outcome depends on the presence of associated anomalies.

In patients in whom the $\mathrm{CM}$ is associated with other abnormalities, the treatment has to be adapted to the single clinical context; however, it appears evident that the Hunt-Lawrence procedure has often been represented an effective and safe option. ${ }^{9}$ Recently a total esophageal gastric dissociation has been proposed by Hattori et al as a treatment option for $\mathrm{CM}$ after the failure of a partial fundoplication procedure. ${ }^{19}$ In our patient, for whom the $\mathrm{CM}$ was an isolated condition, the Hunt-Lawrence procedure was effective in confirming the validity of this technique also when the main problem is not the microgastria per se but altered gastric empting responsible for the patient's problems.

In conclusion, the clinical features of $\mathrm{CM}$ with dumping syndrome correspond to those after gastric resection. In the case reported here Hunt-Lawrence procedure was effective in the treatment of dumping syndrome.

Conflict of Interest

The authors have no conflict of interest to declare.

\section{References}

1 Schulz RD, Niemann F. [Congenital microgastria occurring in combination with skeletal malformations new syndrome] [in German]. Helv Paediatr Acta 1971;26(02):185-191

2 Vasas P, Mudan SS, Akle CA. Congenital microgastria with limb defect combined with megaduodenum: case report and review of literature. Indian J Surg 2011;73(02):122-124

3 Dicken BJ, Novotny NM, Breckler FD, Yim JP, Rescorla FJ. Use of the Hunt-Lawrence pouch in congenital microgastria-a report of 2 cases. J Pediatr Surg 2010;45(11):2238-2240

4 Anderson KD, Guzzetta PC. Treatment of congenital microgastria and dumping syndrome. J Pediatr Surg 1983;18(06): 747-750

5 Shepherd P, Smeulders N, Coleman AH, Chitty LS. Congenital microgastria: a rare cause of failure to visualise the fetal stomach. Prenat Diagn 2011;31(10):1010-1012

6 Menon P, Rao KL, Cutinha HP, Thapa BR, Nagi B. Gastric augmentation in isolated congenital microgastria.J Pediatr Surg 2003;38(10):E4-E6

7 Gorman B, Shaw DG. Congenital microgastria. Br J Radiol 1984; 57(675):260-262

8 Jones VS, Cohen RC. An eighteen year follow-up after surgery for congenital microgastria-case report and review of literature. J Pediatr Surg 2007;42(11):1957-1960

9 Ramos CT, Moss RL, Musemeche CA. Microgastria as an isolated anomaly. J Pediatr Surg 1996;31(10):1445-1447

10 Murray KF, Lillehei CW, Duggan C. Congenital microgastria: treatment with transient jejunal feedings. J Pediatr Gastroenterol Nutr 1999;28(03):343-345

11 Hoehner JC, Kimura K, Soper RT. Congenital microgastria. J Pediatr Surg 1994;29(12):1591-1593

12 Moulton SL, Bouvet M, Lynch FP. Congenital microgastria in a premature infant. J Pediatr Surg 1994;29(12):1594-1595

13 Laurie DE, Wakeling EL. Congenital microgastria in association with Pierre-Robin sequence. Clin Dysmorphol 2008;17(02): $143-144$

14 Scott HW Jr, Gobbel WG Jr, Law DH IV. Clinical experience with a jejunal pouch (Hunt-Lawrence) as a substitute stomach after total gastrectomy. Surg Gynecol Obstet 1965;121(06): 1231-1242

15 Bizzarri C, Cervoni M, Crea F, et al. Dumping syndrome: an unusual cause of severe hyperinsulinemic hypoglycemia in neurologically impaired children with gastrostomy. Minerva Pediatr 2011;63(01):67-71 
e20 Severe Dumping Syndrome Treated by Hunt-Lawrence Pouch Filisetti et al.

16 Gariepy CE, Mousa H. Clinical management of motility disorders in children. Semin Pediatr Surg 2009;18(04):224-238

17 Calabria AC, Charles L, Givler S, De León DD. Postprandial hypoglycemia in children after gastric surgery: clinical characterization and pathophysiology. Horm Res Paediatr 2016; 85(02):140-146
18 Johnson LP, Jesseph JE. Evidence for a humoral etiology of the dumping syndrome. Surg Forum 1961;12:316-317

19 Hattori K, Bvulani B, Numanoglu A, Cox SG, Millar AJ. Total Esophageal gastric dissociation for the failed antireflux procedure in a child with microgastria. European J Pediatr Surg Rep 2016; 4(01):6-9 